\title{
Matching with Preferences over Colleagues Solves Classical Matching
}

\author{
Scott Duke Kominers ${ }^{* 1}$ \\ Department of Economics, Harvard University and Harvard Business School
}

\begin{abstract}
In this note, we demonstrate that the problem of "many-to-one matching with (strict) preferences over colleagues" is actually more difficult than the classical many-to-one matching problem, "matching without preferences over colleagues." We give an explicit reduction of any problem of the latter type to a problem of the former type. This construction leads to the first algorithm which finds all stable matchings in the setting of "matching without preferences over colleagues," for any set of preferences. Our construction directly extends to generalized matching settings.
\end{abstract}

Key words: many-to-one matching, stability, matching algorithms.

JEL: C78, D71

\section{Introduction}

The literature on two-sided matching markets has grown substantially in recent years following high-profile applications of matching in labor markets and school choice programs. $^{2}$ Most practical applications of matching mechanisms require stable matchings, matchings for which no set of agents (strictly) prefers to match among itself than to be matched to its assigned set of partners. However, methods for finding the set of all stable matchings have proven difficult to obtain, even in classical matching settings. ${ }^{3}$

\footnotetext{
*Corresponding author.

Email addresses: kominers@ fas . harvard.edu (Scott Duke Kominers), skominers@hbs .edu (Scott Duke Kominers)

URL: http: / /www. scottkom.com/ (Scott Duke Kominers)

${ }^{1}$ Baker Library 420C, Harvard Business School, Soldiers Field, Boston, MA 02163

${ }^{2}$ See Roth and Sotomayor (1990) for a survey of the pre-1990 theory of matching, and see Roth (2008) for an updated account, as well as references for historical and recent applications of matching.

${ }^{3}$ Three notable exceptions are the algorithms of McVitie and Wilson (1971), Irving and Leather (1986), and Martínez et al. (2004). The first two of these algorithms find all stable matchings in a one-to-one matching market; the last algorithm finds all stable matchings in a classical many-to-many matching market when agents' preferences are substitutable.

Instead of seeking the complete set of stable matchings, much recent work has sought "compromises," stable matchings which fairly balance the concerns of both sides of the market. Teo and Sethuraman (1998), Fleiner (2003), Sethuraman et al. (2006), Klaus and Klijn (2006), and Schwarz and Yenmez (2008) proved
} 
In this note, we give the first algorithm for finding the complete set of stable matchings in any (classical) many-to-one matching market. ${ }^{4}$ This algorithm follows from a reduction of the classical matching problem to "matching with (strict) preferences over colleagues," a nonclassical matching problem which has recently been solved by Echenique and Yenmez (2007).

\subsection{Our Results}

In their work, Echenique and Yenmez (2007) informally claimed the problem of finding stable matchings in the setting of "matching with (strict) preferences over colleagues," to be somehow more difficult than the problem of finding stable matchings in the classical many-to-one matching setting. ${ }^{5}$

We demonstrate in Section 3 that this proposition is true: every classical many-toone matching problem may be solved in the setting of Echenique and Yenmez (2007). This result is surprising because the addition of strict preferences over colleagues to a classical matching problem amounts to breaking a large number of indifferences ${ }^{6}$ and such tie-breaking can affect the set of stable matchings. ${ }^{7}$ Our methods are constructive and therefore give rise to an algorithm, discussed in Section 3.3, for finding all stable many-to-one matchings in the classical setting. This construction is nontrivial-as we discuss in Section 4, the relationship between the two matching settings is more subtle than Echenique and Yenmez (2007) appear to have observed. All of our results extend to generalized matching settings; in particular, they may be applied in the setting of many-to-one matching with contracts.

\subsection{Survey of the Related Literature}

In their seminal paper on two-sided matching, Gale and Shapley (1962) constructively demonstrated that stable matchings always exist in one-to-one and many-to-one (respectively, "marriage" and "college admissions") matching markets when agents have strict, responsive preferences. However, their algorithm only finds extremal matchings, those matchings which are most preferred by agents on one side of the market.

Extremal matchings may not be reasonable outcomes for practical mechanism design. Moreover, preferences may not be responsive in practice, even in otherwise classical matching problems. For these problems and for more general matching settings such as those of "matching with contracts" (Hatfield and Milgrom (2005)), the set

the existence of median stable matchings in a variety of settings; these matchings represent one natural realization of the idea of a compromise matching.

${ }^{4}$ We allow general preferences. In particular, we do not require that participants in the market have responsive preferences.

${ }^{5}$ Of course, these two problems coincide in the setting of one-to-one matching, since there no agent has colleagues.

${ }^{6}$ In the original problem, each student $s$ is indifferent between attending college $c$ with colleague set $S_{1}$ and attending college $c$ with colleague set $S_{2} \neq S_{1}$. Since the framework of Echenique and Yenmez (2007) requires strict preferences, student $s$ must be assigned a strict preference over $S_{1}$ and $S_{2}$; thus, tie-breaking is required.

${ }^{7}$ Abdulkadìroğlu et al. (Forthcoming) discussed how tie-breaking creates "artificial stability constraints." 
of stable matchings may be empty and may be unusually structured even when it is nonempty. ${ }^{8}$ Thus, it is desirable to understand the complete set of stable matchings.

Departing from the prior work on many-to-one matching, ${ }^{9}$ Dutta and Massó (1997) introduced "matching with (strict) preferences over colleagues," 10 a nonclassical manyto-one matching model in which colleges are matched to students who have strict preferences over both their assigned colleges and their student colleagues. ${ }^{11}$ They showed that the set of core matchings in this problem is always nonempty when student preferences are college-lexicographic, that is, when students care first about their colleges and then about their colleagues within that college. ${ }^{12}$ Additionally, they showed that the core is also nonempty in a large class of colleague-lexicographic problems with couples. Revilla (2007) extended these results to the case of general preferences over colleagues and introduced new conditions guaranteeing the existence of core matchings. In recent work, Pycia (2007) found a condition both sufficient and-in some sense-necessary for the existence of stable matchings in the presence of both peer effects and complementarities between agents. ${ }^{13}$

Recently, Echenique and Yenmez (2007) solved the Dutta and Massó (1997) "matching with (strict) preferences over colleagues" model, obtaining an algorithm which finds all stable matchings in this setting. ${ }^{14}$ This result was surprising, in part because no algorithm for finding all stable matchings in the classical many-to-one matching problem (with unrestricted preferences) was available. As we discuss in Section 3.3, the Echenique and Yenmez (2007) algorithm plays a key role in our results. Specifically, our algorithm for finding all stable matchings in classical many-to-one matching settings is obtained by combining our reduction (presented in Section 3) with the algorithm of Echenique and Yenmez (2007).

\section{Model, Notations, and Conventions}

There is a set $C$ of colleges and a set $S$ of students. We consider two types of college-student matching problems in this paper: matchings without and with strict

\footnotetext{
${ }^{8}$ Hatfield and Kojima (2007) have demonstrated that extremal matchings need not exist in a matching market with contracts, even when the set of stable matchings is nonempty.

${ }^{9}$ Most work on many-to-one matching (e.g., Gale and Shapley (1962), Kelso and Crawford (1982), Roth (1985), and Hatfield and Milgrom (2005)) assumes that agents on one side of the market only have preferences over agents (or sets of agents) on the opposite side of the market.

${ }^{10}$ We add the "strict" qualifier to the title "matching with preferences over colleagues" which has been used in the literature, in order to emphasize the importance of strict preferences in the "matching with preferences over colleagues" model.

${ }^{11}$ The colleagues of a student $s$ are the students who are assigned to the same college as $s$.

${ }^{12}$ Dutta and Massó (1997), Revilla (2007), and Pycia (2007) actually used the language of "workers" and "firms," rather than that of "students" and "colleges." However, our work is most closely related to that of Echenique and Yenmez (2007), who phrased the many-to-one matching problem as a "college admissions" problem. Thus, we use the college admissions language uniformly throughout.

${ }^{13}$ Dimitrov and Lazarova (2008) took this work a step further, viewing "matching with (strict) preferences over colleagues" as a weakened model of coalition formation. They presented a generalized to a situation in which coalition formation is allowed on both sides of the market and coalitions themselves can "match" together.

${ }^{14}$ Kojima (2007) extended the method of Echenique and Yenmez (2007) to obtain an algorithm for finding all stable one-to-one matchings in the presence of couple constraints.
} 
preferences over colleagues, respectively called classical and nonclassical matchings. ${ }^{15}$

In both types of matching problems, each college $c \in C$ is assumed to have a strict, transitive, and complete preference relation $P_{c}$ over the set $\wp(S)$ of subsets of $S$. In classical matching problems, each student $s \in S$ is assumed to have a strict, transitive, and complete preference relation $P_{s}$ over the set $C \cup\{\emptyset\}$; such a preference relation is called a classical preference relation. By contrast, in nonclassical matching problems, students $s \in S$ have strict, transitive, and complete preference relations $\bar{P}_{s}$ over the sets $\left(C \times \wp(S)_{s}\right) \cup\{(\emptyset, \emptyset)\}$, where $\wp(S)_{s}=\left\{S^{\prime} \in \wp(S): s \in S^{\prime}\right\}$; these relations are called nonclassical preference relations. For notational convenience, we write only the individually rational component of a preference relation, so that if being unmatched is preferable to being matched to some $\chi$, then $\chi$ does not appear in the preference relation.

The nonstrict part of a preference relation $P_{i}$ is denoted by $R_{i}$. For a preference relation $P_{c}$ over elements of $\wp(S)$, we define the s-restriction $\left.P_{c}\right|_{s}$ of $P_{c}$ to be the preference relation on $\wp(S)_{s}$ induced by $P_{c}$. That is, if $S_{1} P_{c} \cdots P_{c} S_{k} P_{c} \emptyset$, and $\left\{j_{1}, \ldots, j_{k^{\prime}}\right\} \subset\{1, \ldots, k\}$ is the set of indices $j$ for which $s \in S_{j}$, with $j_{1}<\cdots<$ $j_{k^{\prime}}$, then the preference relation $\left.P_{c}\right|_{s}$ is given by $\left.\left.S_{j_{1}} P_{c}\right|_{s} \cdots P_{c}\right|_{s} S_{j_{k^{\prime}}} .{ }^{16}$

We say that a mapping $\mu$ supported on $C \cup S$ is a classical matching if

1. $\mu(c) \in \wp(S)$ for all $c \in C$,

2. $\mu(s) \in C \cup\{\emptyset\}$ for all $s \in S$, and

3. $s \in \mu(c)$ if and only if $\mu(s)=c$.

Similarly, a mapping $\nu$ on $C \cup S$ is said to be a nonclassical matching if

1. $\nu(c) \in \wp(S)$ for all $c \in C$,

2. $\nu(s) \in\left(C \times \wp(S)_{s}\right) \cup\{(\emptyset, \emptyset)\}$ for all $s \in S$,

3. $\nu(s)=(c, \nu(c))$ whenever $s \in \nu(c)$, and

4. $\nu(c)=S^{\prime}$ whenever $\left(c, S^{\prime}\right)=\nu(s)$ for some $s \in S$.

A classical preference profile (resp. nonclassical preference profile) is a collection of preference relations $\left\{P_{c}\right\}_{c \in C} \cup\left\{P_{s}\right\}_{s \in S}$ associated to a classical matching problem (resp. a nonclassical matching problem). We treat a preference profile as a map from $S \cup C$ to the appropriate set of preference relations.

For a classical matching $\mu$ and classical preference profile $\mathcal{P}$, a triple $\left(C^{\prime}, S^{\prime}, \mu^{\prime}\right)$ (with $C^{\prime} \subset C, S^{\prime} \subset S$, and $\mu^{\prime}$ a classical matching) such that

1. $C^{\prime} \cup S^{\prime} \neq \emptyset$,

2. for all $c \in C^{\prime}, \mu^{\prime}(c) \in \wp\left(S^{\prime}\right)$,

3. for all $s \in S^{\prime}, \mu^{\prime}(s) \in C^{\prime} \cup\{\emptyset\}$,

4. for all $i \in C^{\prime} \cup S^{\prime}, \mu^{\prime}(i) R_{i} \mu(i)$ (where $P_{i}=\mathcal{P}(i)$ ), and

\footnotetext{
${ }^{15}$ Of course, there are multiple nonclassical many-to-one matching models. However, in this paper we will only address one, the model of "matching with (strict) preferences over colleagues" introduced by Dutta and Massó (1997) and studied by Echenique and Yenmez (2007).

${ }^{16}$ For a concrete example: if $\left\{s_{1}, s_{2}\right\} P_{c}\left\{s_{1}\right\} P_{c}\left\{s_{2}\right\} P_{c} \emptyset$, then the preference relation $\left.P_{c}\right|_{s_{2}}$ is given by $\left.\left\{s_{1}, s_{2}\right\} P_{c}\right|_{s_{2}}\left\{s_{2}\right\}$.
} 
5. for some $i \in C^{\prime} \cup S^{\prime}, \mu^{\prime}(i) P_{i} \mu(i)$ (where $P_{i}=\mathcal{P}(i)$ ),

is called a $\mathcal{P}$-blocking triple for $\mu$. A classical matching $\mu$ is said to be stable with respect to a classical preference profile $\mathcal{P}$ if there exists no $\mathcal{P}$-blocking triple for $\mu$. Analogously, for a nonclassical matching $\nu$ and nonclassical preference profile $\overline{\mathcal{P}}$, a triple $\left(C^{\prime}, S^{\prime}, \nu^{\prime}\right)$ (with $C^{\prime} \subset C, S^{\prime} \subset S$, and $\nu^{\prime}$ a nonclassical matching) is a $\overline{\mathcal{P}}$ blocking triple for $\nu$ if

1. $C^{\prime} \cup S^{\prime} \neq \emptyset$,

2. for all $c \in C^{\prime}, \nu^{\prime}(c) \in \wp\left(S^{\prime}\right)$,

3. for all $s \in S^{\prime}, \nu^{\prime}(s) \in\left(C^{\prime} \times \wp\left(S^{\prime}\right)_{s}\right) \cup\{(\emptyset, \emptyset)\}$,

4. for all $i \in C^{\prime} \cup S^{\prime}, \mu^{\prime}(i) \bar{R}_{i} \mu(i)$ (where $\bar{P}_{i}=\overline{\mathcal{P}}(i)$ ), and

5. for some $i \in C^{\prime} \cup S^{\prime}, \mu^{\prime}(i) \bar{P}_{i} \mu(i)$ (where $\bar{P}_{i}=\overline{\mathcal{P}}(i)$ ).

A nonclassical matching $\nu$ is said to be stable with respect to a nonclassical preference profile $\overline{\mathcal{P}}$ if there exists no $\overline{\mathcal{P}}$-blocking triple for $\nu$.

\section{Reducing Classical Matching to Nonclassical Matching}

In this section, we show that, for any classical preference profile $\mathcal{P}$, there is an associated nonclassical preference profile $\overline{\mathcal{P}}(\mathcal{P})$ such that the nonclassical matchings stable with respect to $\overline{\mathcal{P}}(\mathcal{P})$ exactly correspond to the classical matchings stable with respect to $\mathcal{P}$.

\subsection{The Construction}

We present an explicit construction of the nonclassical preference profile $\overline{\mathcal{P}}(\mathcal{P})$. In Section 3.1.1, we provide a simple demonstrative example of this construction. We prove the correspondence between the nonclassical matchings stable with respect to $\overline{\mathcal{P}}(\mathcal{P})$ and the classical matchings stable with respect to $\mathcal{P}$ in Section 3.2 and then revisit our example in Section 3.2.1. Then, in Section 3.3, we discuss how combining our construction and the results of Echenique and Yenmez (2007) yields an algorithm for finding all matchings stable with respect to $\mathcal{P}$.

Construction 1. Let $\mathcal{P}$ be a classical preference profile. Define a nonclassical preference profile $\overline{\mathcal{P}}(\mathcal{P})$ by the following rules:

1. Set $(\overline{\mathcal{P}}(\mathcal{P}))(c)=\mathcal{P}(c)$ for all $c \in C$.

2. For any $s \in S$, let $P_{s}=\mathcal{P}(s)$, and write this preference relation in the form $c_{1} P_{s} \cdots P_{s} c_{k} P_{s} \emptyset .{ }^{17}$ Then, for each college $c_{j}(1 \leq j \leq k)$, set $P_{c_{j}}=\mathcal{P}\left(c_{j}\right)$ and write the preference relation $\left.P_{c_{j}}\right|_{s}$ as

$$
\left.\left.S_{j, 1} P_{c_{j}}\right|_{s} \cdots P_{c_{j}}\right|_{s} S_{j, \ell_{j}}
$$

\footnotetext{
${ }^{17}$ Here, $k=k(s)$ is the number of colleges $c_{i} \in C$ acceptable to $s$. Without loss of generality, we have assumed $k \geq 1$, since if $k=0$ then $s$ does not need to be matched.
} 
Finally, define the preference relation $\bar{P}_{s}=(\overline{\mathcal{P}}(\mathcal{P}))(s)$ by

$$
\left(c_{1}, S_{1,1}\right) \bar{P}_{s} \cdots \bar{P}_{s}\left(c_{1}, S_{1, \ell_{1}}\right) \bar{P}_{s} \cdots \bar{P}_{s}\left(c_{k}, S_{k, 1}\right) \bar{P}_{s} \cdots \bar{P}_{s}\left(c_{k}, S_{k, \ell_{k}}\right) \bar{P}_{s}(\emptyset, \emptyset) .{ }^{18}
$$

Intuitively, this construction exactly aligns students' preferences over colleagues with colleges' preferences over sets of students.

\subsubsection{A Simple Example}

To clarify Construction 1, we discuss a simple illustrative example. Suppose that $C=\left\{c_{1}, c_{2}\right\}, S=\left\{s_{1}, s_{2}\right\}$, and write $P_{i}=\mathcal{P}(i)$ for $i \in C \cup S$. Suppose further that the preference relations $P_{i}$ are given by

$$
\left\{s_{1}, s_{2}\right\} P_{c_{1}}\left\{s_{1}\right\} P_{c_{1}}\left\{s_{2}\right\} P_{c_{1}} \emptyset, \quad\left\{s_{1}\right\} P_{c_{2}}\left\{s_{2}\right\} P_{c_{2}} \emptyset, \quad c_{1} P_{s_{1}} c_{2} P_{s_{1}} \emptyset, \quad c_{2} P_{s_{2}} \emptyset .
$$

Then, if we write $\bar{P}_{i}=(\overline{\mathcal{P}}(\mathcal{P}))(i)$, Construction 1 gives that

$$
\begin{gathered}
\left\{s_{1}, s_{2}\right\} \bar{P}_{c_{1}}\left\{s_{1}\right\} \bar{P}_{c_{1}}\left\{s_{2}\right\} \bar{P}_{c_{1}} \emptyset, \quad\left\{s_{1}\right\} \bar{P}_{c_{2}}\left\{s_{2}\right\} \bar{P}_{c_{2}} \emptyset \\
\left(c_{1},\left\{s_{1}, s_{2}\right\}\right) \bar{P}_{s_{1}}\left(c_{1},\left\{s_{1}\right\}\right) \bar{P}_{s_{1}}\left(c_{2},\left\{s_{1}\right\}\right) \bar{P}_{s_{1}}(\emptyset, \emptyset), \quad\left(c_{2},\left\{s_{2}\right\}\right) \bar{P}_{s_{2}}(\emptyset, \emptyset) .
\end{gathered}
$$

Following the intuition described in the previous section, we observe that, under the preferences defined by $\overline{\mathcal{P}}(\mathcal{P})$, a student $s$ prefers a pair $\left(c, S_{1}\right) \in\left(C \times \wp(S)_{s}\right) \cup\{(\emptyset, \emptyset)\}$ to another pair $\left(c, S_{2}\right) \in\left(C \times \wp(S)_{s}\right) \cup\{(\emptyset, \emptyset)\}$ if and only if $c$ prefers $S_{1}$ to $S_{2}$. Thus, student and college preferences over subsets of students are aligned.

\subsection{The Matching Correspondence}

In order to relate the stable matchings of the preference profiles $\mathcal{P}$ and $\overline{\mathcal{P}}(\mathcal{P})$, we will need a bit more terminology. We define the college projection $\operatorname{proj}_{c}\left(\left(c^{\prime}, S^{\prime}\right)\right)$ of a pair $\left(c^{\prime}, S^{\prime}\right) \in C \times \wp(S)_{s}$ by $\operatorname{proj}_{c}\left(\left(c^{\prime}, S^{\prime}\right)\right)=c^{\prime}$. For a nonclassical matching $\nu$, we define the classical matching $\mu_{\nu}$ associated to $\nu$ by

$$
\mu_{\nu}(c)=\nu(c) \quad(\forall c \in C), \quad \mu_{\nu}(s)=\operatorname{proj}_{c}(\nu(s)) \quad(\forall s \in S) .{ }^{19}
$$

We may now state our main result.

Proposition 1. For a classical preference profile $\mathcal{P}$, let $\overline{\mathcal{P}}(\mathcal{P})$ be as defined in Construction 1. A classical matching $\mu$ is stable with respect to $\mathcal{P}$ if and only if there is a nonclassical matching $\nu$ stable with respect to $\overline{\mathcal{P}}(\mathcal{P})$ such that $\mu_{\nu} \equiv \mu$.

Proof. For all $i \in C \cup S$, we write $P_{i}=\mathcal{P}(i)$ and $\bar{P}_{i}=(\overline{\mathcal{P}}(\mathcal{P}))(i)$. For the "if" direction, we consider some nonclassical matching $\nu$ stable with respect to the preference profile $\overline{\mathcal{P}}(\mathcal{P})$ and suppose for the sake of contradiction that $\mu_{\nu}$ is not stable with respect to $\mathcal{P}$. Then, there is some $\mathcal{P}$-blocking triple $\left(C^{\prime}, S^{\prime}, \mu^{\prime}\right)$ for $\mu_{\nu}$. Now, for any $c \in C^{\prime}$

\footnotetext{
${ }^{18}$ In this construction, the preference relation $P_{s}$ defined by (2) is a well-defined nonclassical preference relation for the student $s$. Indeed, $s \in S_{j, \ell}$ for all $1 \leq j \leq k$ and $1 \leq \ell \leq \ell_{i}$ by the definition (1) and no pair $\left(c_{j}, S_{j, \ell}\right)$ is repeated in (2) because the preference relations $P_{c_{j}}$ are well-defined.

${ }^{19}$ It is clear that this definition actually defines a classical matching $\mu_{\nu}$.
} 
and $s \in \mu^{\prime}(c)$, we know that $\mu^{\prime}(c) R_{c} \mu_{\nu}(c)$ and $\mu^{\prime}(s) R_{s} \mu_{\nu}(s) .{ }^{20}$ By the construction of $\bar{P}_{s}$, we then have

$$
\left(c, \mu^{\prime}(c)\right) \bar{R}_{s}\left(\mu_{\nu}(s), \mu_{\nu}\left(\mu_{\nu}(s)\right)\right),
$$

for any $c \in C^{\prime}$ and $s \in \mu^{\prime}(c)$. Moreover, the relationship (3) is strict for at least one $s \in S^{\prime}$, since at least one of the relationships

$$
\left\{\mu^{\prime}(c) R_{c} \mu_{\nu}(c)\right\}_{c \in C^{\prime}} \quad \text { or } \quad\left\{\mu^{\prime}(s) R_{s} \mu_{\nu}(s)\right\}_{s \in S^{\prime}}
$$

is strict. But then, it follows quickly that the triple $\left(C^{\prime}, S^{\prime}, \nu^{\prime}\right)$ with $\nu^{\prime}$ defined by

$$
\nu^{\prime}(i)= \begin{cases}\mu^{\prime}(i) & i \in C^{\prime} \\ \left(\mu^{\prime}(i), \mu^{\prime}\left(\mu^{\prime}(i)\right)\right) & i \in S^{\prime} \\ \nu(i) & i \in(C \cup S) \backslash\left(C^{\prime} \cup S^{\prime}\right)\end{cases}
$$

is a $\overline{\mathcal{P}}(\mathcal{P})$-blocking triple for $\nu$, contradicting the stability of $\nu$.

For the "only if" direction, it suffices to observe that the nonclassical matching $\nu$ defined by

$$
\nu(i)= \begin{cases}\mu(i) & i \in C, \\ (\mu(i), \mu(\mu(i))) & i \in S,\end{cases}
$$

is stable with respect to $\overline{\mathcal{P}}(\mathcal{P})$. This follows directly from the stability of $\mu$ and the construction of $\overline{\mathcal{P}}(\mathcal{P})$, so we omit the details.

\subsubsection{Revisiting the Simple Example}

We now return to the example presented in Section 3.1.1 and observe that the unique nonclassical matching $\nu$ stable with respect to $\overline{\mathcal{P}}(\mathcal{P})$ is given by

$$
\nu(i)= \begin{cases}\left\{s_{1}\right\} & i=c_{1}, \\ \left\{s_{2}\right\} & i=c_{2}, \\ \left(c_{1},\left\{s_{1}\right\}\right) & i=s_{1}, \\ \left(c_{2},\left\{s_{2}\right\}\right) & i=s_{2} .\end{cases}
$$

This nonclassical matching $\nu$ restricts to the unique classical matching $\mu$ stable with respect to $\mathcal{P}$ :

$$
\mu_{\nu}(i) \equiv \mu(i)= \begin{cases}\left\{s_{1}\right\} & i=c_{1} \\ \left\{s_{2}\right\} & i=c_{2} \\ c_{1} & i=s_{1} \\ c_{2} & i=s_{2}\end{cases}
$$

\footnotetext{
${ }^{20}$ Here, we use the convention that $\emptyset \in C^{\prime}$ if the set $C^{\prime}$ is empty.
} 


\subsection{Finding All Stable (Classical) Matchings}

Echenique and Yenmez (2007) presented an algorithm finding all nonclassical matchings stable with respect to a given nonclassical preference profile $\overline{\mathcal{P}}$. Their approach uses a fixed-point characterization of stability-machinery which is not yet known to exist in the setting of classical many-to-one matching. ${ }^{21}$

Our Proposition 1 shows that, in theory, it is possible to find all classical matchings stable with respect to a classical preference profile $\mathcal{P}$ by applying the algorithm of Echenique and Yenmez (2007) to $\overline{\mathcal{P}}(\mathcal{P})$. We must note, however, that such an operation is likely to be computationally costly. Indeed, if $|\hat{\mathcal{P}}|$ denotes the length of the longest preference relation in a preference profile $\hat{\mathcal{P}}$, then $|\overline{\mathcal{P}}(\mathcal{P})|=O\left(|\mathcal{P}|^{2}\right)$ by construction. The size of the input data when running the Echenique and Yenmez (2007) algorithm on $\overline{\mathcal{P}}(\mathcal{P})$ is therefore of the same order as the running time of the deferred acceptance algorithm run on $\mathcal{P} .^{22}$

\subsection{Extensions}

Our Construction 1 is essentially independent of the domains of the input preference relations. Thus, it admits simple generalizations to more complex matching settings. Most notably, an analogous construction may be used in the Hatfield and Milgrom (2005) setting of matching with contracts to reduce a classical "matching with contracts" problem to a problem of "matching with contracts and preferences over colleagues' contracts."

\section{Variants of Construction 1}

Although Proposition 1 shows that the problem of finding stable nonclassical matchings is more difficult than the problem of finding classical matchings, this relationship is rather subtle. ${ }^{23}$ As we mentioned in Section 1, the process of constructing a nonclassical preference profile $\overline{\mathcal{P}}^{\prime}(\mathcal{P})$ from a classical preference profile $\mathcal{P}$ entails a large amount of tie-breaking; if not executed carefully, such tie-breaking can affect the set of stable matchings.

\subsection{An Illustrative Example}

For an example of the effects of indiscriminate tie-breaking, we suppose that

$$
C=\{c\}, \quad S=\left\{s_{1}, s_{2}\right\},
$$

\footnotetext{
${ }^{21}$ Such a characterization does exist in classical one-to-one matching settings; this result is originally due to Adachi (2000) and also arises in the one-to-one matching specialization of the results of Echenique and Yenmez (2007).

${ }^{22}$ Unfortunately, we are unable to provide full complexity analysis of this method for finding stable classical matchings, since Echenique and Yenmez (2007) did not completely analyze the running time of their algorithm.

${ }^{23}$ Technically, Proposition 1 only shows that the problem of finding stable nonclassical matchings is weakly more difficult than the problem of finding classical matchings. However, it is clear that there are nonclassical matching problems which do not correspond to classical matching problems.
} 
and that the classical preference profile $\mathcal{P}$ is given by

$$
\left\{s_{1}, s_{2}\right\} P_{c}\left\{s_{1}\right\} P_{c} \emptyset, \quad c P_{s_{1}} \emptyset, \quad c P_{s_{2}} \emptyset,
$$

where $P_{i}=\mathcal{P}(i)$ for all $i \in C \cup S$. In order to construct a nonclassical preference profile from $\mathcal{P}$ which respects the ordering of preferencs in $\mathcal{P},{ }^{24}$ we must break one indifference: that of $s_{1}$ between the options $\left(c,\left\{s_{1}, s_{2}\right\}\right)$ and $\left(c,\left\{s_{1}\right\}\right)$.

Applying Construction 1 to $\mathcal{P}$ gives the following nonclassical preference profile $\overline{\mathcal{P}}(\mathcal{P})$ :

$$
\left\{s_{1}, s_{2}\right\} \bar{P}_{c}\left\{s_{1}\right\} \bar{P}_{c} \emptyset, \quad\left(c,\left\{s_{1}, s_{2}\right\}\right) \bar{P}_{s_{1}}\left(c,\left\{s_{1}\right\}\right) \bar{P}_{s_{1}}(\emptyset, \emptyset), \quad\left(c,\left\{s_{1}, s_{2}\right\}\right) \bar{P}_{s_{2}}(\emptyset, \emptyset)
$$

(where $\bar{P}_{i}=(\overline{\mathcal{P}}(\mathcal{P}))(i)$ ). The unique matching $\nu$ stable with respect to $\overline{\mathcal{P}}(\mathcal{P})$ is

$$
\nu(i)= \begin{cases}\left\{s_{1}, s_{2}\right\} & i=c \\ \left(c,\left\{s_{1}, s_{2}\right\}\right) & i \in\left\{s_{1}, s_{2}\right\}\end{cases}
$$

If, instead, the indifference of $s_{1}$ between $\left(c,\left\{s_{1}, s_{2}\right\}\right)$ and $\left(c,\left\{s_{1}\right\}\right)$ is broken differently, then a new nonclassical preference profile $\overline{\mathcal{P}}^{\prime}(\mathcal{P})$ is obtained:

$$
\left\{s_{1}, s_{2}\right\} \bar{P}_{c}^{\prime}\left\{s_{1}\right\} \bar{P}_{c}^{\prime} \emptyset, \quad\left(c,\left\{s_{1}\right\}\right) \bar{P}_{s_{1}}^{\prime}\left(c,\left\{s_{1}, s_{2}\right\}\right) \bar{P}_{s_{1}}^{\prime}(\emptyset, \emptyset), \quad\left(c,\left\{s_{1}, s_{2}\right\}\right) \bar{P}_{s_{2}}^{\prime}(\emptyset, \emptyset)
$$

(where $\bar{P}_{i}^{\prime}=\left(\overline{\mathcal{P}}^{\prime}(\mathcal{P})\right)(i)$ ). There are two matchings stable with respect to $\overline{\mathcal{P}}^{\prime}(\mathcal{P}): \nu$ and $\nu^{\prime}$, where

$$
\nu^{\prime}(i)= \begin{cases}\left\{s_{1}\right\} & i=c, \\ \left(c,\left\{s_{1}\right\}\right) & i=s_{1}, \\ (\emptyset, \emptyset) & i=s_{2} .\end{cases}
$$

While $\mu_{\nu}$ is the unique matching stable with respect to $\mathcal{P}$, it is clear that $\mu_{\nu^{\prime}} \not \equiv \mu_{\nu}$ is not stable with respect to $\mathcal{P}$. Thus, we see that $\overline{\mathcal{P}}(\mathcal{P})$ is the only nonclassical preference profile which both respects the preference ordering of $\mathcal{P}$ and yields a bijective stable matching correspondence.

\subsection{Discussion}

The example just presented demonstrates that a nonclassical preference profile $\overline{\mathcal{P}}^{\prime}(\mathcal{P}) \not \equiv \overline{\mathcal{P}}(\mathcal{P})$ constructed from a classical preference profile $\mathcal{P}$ may not admit a bijective stable matching correspondence.

Nonetheless, we may obtain the reverse implication of Proposition 1 under relatively intuitive conditions which are weaker than the requirement of the full form of Construction 1. This is formalized in the following proposition.

Proposition 2. Let $\mathcal{P}$ be a classical preference profile, and let $\overline{\mathcal{P}}^{\prime}(\mathcal{P})$ be a nonclassical preference profile such that

\footnotetext{
${ }^{24}$ It is clear that the set of stable matchings may shrink if the ordering of preferences in $\mathcal{P}$ is not respected. However, as we show in Proposition 2, the set of stable matchings may not shrink if the ordering of preferences in $\mathcal{P}$ is respected.
} 
1. $\left(\overline{\mathcal{P}}^{\prime}(\mathcal{P})\right)(c)=\mathcal{P}(c)$ for all $c \in C$,

2. if $c P_{s} \emptyset$ and $S^{\prime} P_{c} \emptyset$ for some $s \in S, c \in C$, and $S^{\prime} \in \wp(S)_{s}$, then $\left(c, S^{\prime}\right) \bar{P}_{s}^{\prime}(\emptyset, \emptyset)$,

3. if $\left(c_{1}, S_{1}\right) \bar{R}_{s}^{\prime}\left(c_{2}, S_{2}\right)$ for some $s \in S, c_{1}, c_{2} \in C$, and $S_{1}, S_{2} \in \wp(S)_{s}$, then $c_{1} R_{s} c_{2}$

where $P_{i}=\mathcal{P}(i)$ and $\bar{P}_{i}^{\prime}=\left(\overline{\mathcal{P}}^{\prime}(\mathcal{P})\right)(i)$ for all $i \in C \cup S$. Then, if $\mu$ is a matching stable with respect to $\mathcal{P}$, the matching $\nu$ defined by

$$
\nu(i)= \begin{cases}\mu(i) & i \in C, \\ (\mu(i), \mu(\mu(i))) & i \in S,\end{cases}
$$

is stable with respect to $\overline{\mathcal{P}}^{\prime}(\mathcal{P})$.

The proof of Proposition 2 is immediate from the fact that the preference profile $\overline{\mathcal{P}}^{\prime}(\mathcal{P})$ respects the preference orderings of $\mathcal{P}$. Indeed, it follows quickly from the conditions of the proposition statement that, if $\left(C^{\prime}, S^{\prime}, \nu^{\prime}\right)$ is a $\left(\overline{\mathcal{P}}^{\prime}(\mathcal{P})\right)$-blocking triple for $\nu$, then $\left(C^{\prime}, S^{\prime}, \mu_{\nu^{\prime}}\right)$ is a $\mathcal{P}$-blocking triple for $\mu$.

Although Proposition 2 recovers the reverse implication of Proposition 1 under general conditions, the forward implication of Proposition 1 need not hold under these conditions. ${ }^{25}$ We might hope for a general classification of the set of constructions alternative to Construction 1 which admit bijective correspondence results akin to Proposition 1. Unfortunately, such a classification appears to be out of reach. When restricted to a particular classical preference profile $\mathcal{P}$, this problem includes (as a subproblem) the question of finding all permutations of the preference relations in $\overline{\mathcal{P}}(\mathcal{P})$ which fix the set of stable matchings ${ }^{26}$; this question is not well-understood, even within the simpler setting of one-to-one matching. ${ }^{27}$

\section{Acknowledgments}

I thank Zachary Abel, Peter Coles, Drew Fudenberg, John Hatfield, Andrea Hawksley, Bettina Klaus, Fuhito Kojima, Alvin Roth, and an anonymous referee for their helpful comments and suggestions.

\footnotetext{
${ }^{25}$ To see this, it suffices to note that the example of Section 4.1 satisfies the hypotheses of Proposition 2.

${ }^{26}$ For many classical preference profiles $\mathcal{P}$, this set of permutations is nonempty. For an example, we consider the classic preference profile $\mathcal{P}$ of Section 3.1.1. If we let $\bar{P}_{i}^{\prime}=\left(\overline{\mathcal{P}}^{\prime}(\mathcal{P})\right)(i)$ be defined by

$$
\begin{gathered}
\left\{s_{1}, s_{2}\right\} \bar{P}_{c_{1}}^{\prime}\left\{s_{1}\right\} \bar{P}_{c_{1}}^{\prime}\left\{s_{2}\right\} \bar{P}_{c_{1}}^{\prime} \emptyset, \quad\left\{s_{1}\right\} \bar{P}_{c_{2}}^{\prime}\left\{s_{2}\right\} \bar{P}_{c_{2}}^{\prime} \emptyset, \\
\left(c_{1},\left\{s_{1}\right\}\right) \bar{P}_{s_{1}}^{\prime}\left(c_{1},\left\{s_{1}, s_{2}\right\}\right) \bar{P}_{s_{1}}^{\prime}\left(c_{2},\left\{s_{1}\right\}\right) \bar{P}_{s_{1}}^{\prime}(\emptyset, \emptyset), \quad\left(c_{2},\left\{s_{2}\right\}\right) \bar{P}_{s_{2}}^{\prime}(\emptyset, \emptyset),
\end{gathered}
$$

then $\overline{\mathcal{P}}^{\prime}(\mathcal{P}) \not \equiv \overline{\mathcal{P}}(\mathcal{P})$ but the unique matching stable with respect to $\overline{\mathcal{P}}^{\prime}(\mathcal{P})$ corresponds (via restriction) to the unique matching stable with respect to $\mathcal{P}$.

${ }^{27}$ In what appears to be the only direct work on this problem, Kuo and Tseng (2005) studied the setting of one-to-one matching. They found sufficient conditions for a permutation of a preference profile $\mathcal{P}$ to preserve the male-optimality of the original male-optimal stable matching.

A presumably related, easier problem seeks sufficient conditions for a preference relation to induce a unique stable matching. Both Eeckhout (2000) and Clark (2006) obtained such conditions in the context of one-to-one matching, but the set of preference domains satisfying these conditions is quite small.
} 


\section{References}

Abdulkadìroğlu, A., Pathak, P., Roth, A., Forthcoming. Strategyproofness versus efficiency in matching with indifferences: Redesigning the NYC high school match. American Economic Review.

Adachi, H., 2000. On a characterization of stable matchings. Economics Letters 68, $43-49$.

Clark, S., 2006. The uniqueness of stable matchings. Contributions to Theoretical Economics 6, Article 8.

Dimitrov, D., Lazarova, E., 2008. Coalitional matchings, Fondazione Eni Enrico Mattei Working Paper 45.2008.

Dutta, B., Massó, J., 1997. Stability of matchings when individuals have preferences over colleagues. Journal of Economic Theory 75, 464-475.

Echenique, F., Yenmez, M. B., 2007. A solution to matching with preferences over colleagues. Games and Economic Behavior 59, 46-71.

Eeckhout, J., 2000. On the uniqueness of stable marriage matchings. Economics Letters $69,1-8$.

Fleiner, T., 2003. A fixed point approach to stable matchings and some applications. Mathematics of Operations Research 28, 103-126.

Gale, D., Shapley, L. S., 1962. College admissions and the stability of marriage. American Mathematical Monthly 69, 9-15.

Hatfield, J., Kojima, F., 2007. Substitutes and stability for matching with contracts, mimeo, Cowles Foundation.

Hatfield, J., Milgrom, P., 2005. Matching with contracts. American Economic Review 95, 913-935.

Irving, R., Leather, P., 1986. The complexity of counting stable marriages. SIAM Journal of Computing 15, 655-667.

Kelso, A. S., Crawford, V. P., 1982. Job matching, coalition formation, and gross substitutes. Econometrica 50, 1483-1504.

Klaus, B., Klijn, F., 2006. Median stable matching for college admissions. International Journal of Game Theory 34, 1-11.

Kojima, F., 2007. Finding all stable matchings with couples, mimeo, Cowles Foundation.

Kuo, R. T., Tseng, S. S., 2005. On the invariance of male optimal stable matching. BIT Numerical Mathematics 40, 592-598. 
Martínez, R., Massó, J., Neme, A., Oviedo, J., 2004. An algorithm to compute the full set of many-to-many stable matchings. Mathematical Social Sciences 47, 187-210.

McVitie, D. G., Wilson, L. B., 1971. The stable marriage problem. Communications of the ACM 14, 486-490.

Pycia, M., 2007. Many-to-one matching with complementarities and peer effects, mimeo, Pennsylvania State University.

Revilla, P., 2007. Many-to-one matching when colleagues matter, Fondazione Eni Enrico Mattei Working Paper 87.2007.

Roth, A. E., 1985. The college admissions problem is not equivalent to the marriage problem. Journal of Economic Theory 36, 277-288.

Roth, A. E., 2008. Deferred acceptance algorithms: history, theory, practice, and open questions. International Journal of Game Theory 36, 537-569.

Roth, A. E., Sotomayor, M. A. O., 1990. Two-sided matching: a study in gametheoretic modeling and analysis. Econometric Society Monographs. Cambridge University Press.

Schwarz, M., Yenmez, M. B., 2008. Median stable matching, mimeo, Stanford University.

Sethuraman, J., Teo, C., Qian, L., 2006. Many-to-one matching: Geometry and fairness. Mathematics of Operations Research 31, 581-596.

Teo, C., Sethuraman, J., 1998. The geometry of fractional stable matchings and its applications. Mathematics of Operations Research 23, 874-891. 